\title{
SOLITARY WAVE MODULATION IN AN ARTERY WITH STENOSIS FILLED WITH A VISCOUS FLUID
}

\author{
${ }^{1}$ Yaan Yee Choy, ${ }^{1}$ Kim Gaik Tay and ${ }^{2}$ Chee Tiong Ong \\ ${ }^{1}$ Department of Mathematics and Statistics, \\ Faculty of Science, Universiti Tun Hussein Onn Malaysia, \\ Technology and Human Development, 86400 Parit Raja, Johor, Malaysia \\ ${ }^{2}$ Department of Mathematical Science, \\ Faculty of Science, Universiti Teknologi Malaysia, 81310 Skudai, Johor, Malaysia
}

Received 2013-07-29, Revised 2013-08-20; Accepted 2013-08-21

\begin{abstract}
In this study, the derivation of mathematical model for the wave modulation through an incompressible viscous fluid contained in a prestressed thin stenosed elastic tube is presented. The artery is assumed to be incompressible, prestressed thin walled elastic tube with a symmetrical stenosis, whereas the blood is considered to be incompressible and Newtonian fluid. By utilizing the nonlinear equations of tube and fluid, the weakly nonlinear wave modulation in such a medium is examined. Employing the reductive perturbation method and considering the long-wave approximation, we showed that the third-order term in the perturbation expansion is governed by the dissipative nonlinear Schrodinger equation with variable coefficient. Our results shown that this type of equation admits a downward bell-shape wave propagates to the right as time increases with decreasing wave amplitude.
\end{abstract}

Keywords: Tube, Newtonian Fluid, Wave Modulation, Nonlinear Schrodinger Equation

\section{INTRODUCTION}

Cholesterol is a sterol which serves principally as a constituent of blood plasma. High concentration of cholesterol in the blood will build up plaque on the inner walls of arteries. Over the time, the decomposition of plaque causes the arteries become less flexible and this leads to the phenomenon of narrowing of the arteries which commonly referred as stenosis. The stenosis will further cause the artherosclerosis which is hardening of the arteries. The artherosclerosis leads the arteries lose their elastic properties and the volume of blood carried through the arteries is reduced. Motivated with the stenosis in the artery, the study of wave propagation in a stenosed artery has been investigated by Kim (2006). Tay et al. (2007) and Gaik and Demiray (2008).
Ravindran and Prasad (1979) were the first contributed to the derivation of mathematical model for the nonlinear wave modulation in an artery. They assumed the blood as an compressible inviscid fluid contained in a thin walled viscoelastic tube under certain simplifying assumptions. By using the method of operators of multiple scales, they shown that the governed equation can be reduced to the Nonlinear Schrodinger (NLS) equation for their mathematical model. The NLS equation is the simplest representative equation describing the self-modulation of one-dimensional monochromatic plane waves in dispersive media. It has a balance between the nonlinearity and dispersion.

In a series of works of Demiray (1997; 1998; 2001; 2003) and his co-workers (Akgun and Demiray, 2000; 2001) conducted since 1997, in which they treated the artery as an incompressible, prestressed either thin elastic,

Corresponding Author: Yaan Yee Choy, Department of Mathematics and Statistics, Faculty of Science, Universiti Tun Hussein Onn Malaysia, Technology and Human Development, 86400 Parit Raja, Johor, Malaysia 
thick elastic or tapered elastic tube contained with an incompressible inviscid or viscous fluid as blood. Then by employing the reductive perturbation method, the modulation of nonlinear waves in the long-wave approximation were studied. They obtained various nonlinear evolution equations of the NLS type equations. In all the works of Demiray $(1997 ; 1998 ; 2001 ; 2003)$ as well as Ravindran and Prasad (1979) they treated the artery as circularly cylindrical long tubes without stenosis.

Recently, the studies of nonlinear waves modulation in an incompressible prestressed thin walled elastic tube with a symmetrical stenosis filled with an incompressible inviscid or viscous fluid, where the approximate equations of inviscid and viscous fluids without boundary condition is used have been carried by Choy et al. (2012) and Kim et al. (2010). By using the reductive perturbation method, in the long-wave approximation, they obtained the NLS equation with variable coefficient and dissipative NLS equation with variable coefficient for the study of inviscid and viscous fluids, respectively.

Nevertheless, the study of nonlinear wave modulation in a prestressed thin elastic tube with a symmetrical stenosis filled with a viscous fluid, where the non approximate equations of viscous fluid with its boundary conditions are used has not been carried out yet in literatures. Therefore, we assumed the artery as an incompressible, prestressed, thin walled elastic tube with a symmetrical stenosis and the blood as an incompressible viscous fluid, a mathematical model for the nonlinear wave modulation in such a composite medium, in the long-wave approximation is developed. Applying the reductive perturbation method, we have shown that the amplitude modulation of this wave is governed by the dissipative NLS equation with variable coefficient. The dissipative term in the dissipative NLS equation with variable coefficient is due to the viscous effect. The presence of stenosis leads to the variable coefficient term in the dissipative NLS equation with variable coefficient. Our results reveal that the downward bell-shaped wave propagates to the right with decreasing wave amplitude as time increases.

\subsection{The Governing Equations}

The derivation of the field equations of a stenosed elastic tube, which is considered to be a model for an artery and a viscous fluid, which is assumed to be a model for blood has been carried out by Kim et al. (2010). By introducing the non-dimensional quantities (Kim et al., 2010) into the field equations of tube and fluid, one obtains the following non-dimensional Equations of tube, $\mathrm{P}_{\mathrm{r}}$ and fluid as below:

$$
\begin{aligned}
& \mathrm{p}_{\mathrm{r}}=\frac{\mathrm{m}}{\lambda_{\mathrm{z}}\left[\lambda_{\theta}-\mathrm{f}+\mathrm{u}\right]} \frac{\partial^{2} \mathrm{u}}{\partial \mathrm{t}^{2}}+\frac{1}{\lambda_{\mathrm{z}}\left[\lambda_{\theta}-\mathrm{f}+\mathrm{u}\right]} \frac{\partial \Sigma}{\partial \lambda_{2}} \\
& -\frac{1}{\lambda_{\mathrm{z}}\left[\lambda_{\theta}-\mathrm{f}+\mathrm{u}\right]} \frac{\partial}{\partial \mathrm{z}}\left\{\frac{\left(-\frac{\partial \mathrm{f}}{\partial \mathrm{z}}+\frac{\partial \mathrm{u}}{\partial \mathrm{z}}\right)}{\Lambda} \frac{\partial \Sigma}{\partial \lambda_{1}}\right\} \\
& \frac{\partial \mathrm{v}}{\partial \mathrm{t}}+\mathrm{v} \frac{\partial \mathrm{v}}{\partial \mathrm{r}}+\mathrm{q} \frac{\partial \mathrm{v}}{\partial \mathrm{z}}+\frac{\partial \overline{\mathrm{p}}}{\partial \mathrm{r}}-\overline{\mathrm{v}}\left[\frac{\partial^{2} \mathrm{v}}{\partial \mathrm{r}^{2}}+\frac{1}{\mathrm{r}} \frac{\partial \mathrm{v}}{\partial \mathrm{r}}-\frac{\mathrm{v}}{\mathrm{r}^{2}}+\frac{\partial^{2} \mathrm{v}}{\partial \mathrm{z}^{2}}\right]=0 \\
& \frac{\partial \mathrm{q}}{\partial \mathrm{t}}+\mathrm{v} \frac{\partial \mathrm{q}}{\partial \mathrm{r}}+\mathrm{q} \frac{\partial \mathrm{q}}{\partial \mathrm{z}}+\frac{\partial \overline{\mathrm{p}}}{\partial \mathrm{z}}-\overline{\mathrm{v}}\left[\frac{\partial^{2} \mathrm{q}}{\partial \mathrm{r}^{2}}+\frac{1}{\mathrm{r}} \frac{\partial \mathrm{q}}{\partial \mathrm{r}}+\frac{\partial^{2} \mathrm{q}}{\partial \mathrm{z}^{2}}\right]=0 \\
& \frac{\partial \mathrm{v}}{\partial \mathrm{r}}+\frac{\mathrm{v}}{\mathrm{r}}+\frac{\partial \mathrm{q}}{\partial \mathrm{z}}=0
\end{aligned}
$$

With the boundary conditions:

$$
\begin{gathered}
\mathrm{p}_{\mathrm{r}}=\left.\left[\left(\overline{\mathrm{p}}_{0}+\overline{\mathrm{p}}\right)-2 \overline{\mathrm{v}} \frac{\partial \mathrm{v}}{\partial \mathrm{r}}+\overline{\mathrm{v}}\left(\frac{\partial \mathrm{v}}{\partial \mathrm{z}}+\frac{\partial \mathrm{q}}{\partial \mathrm{r}}\right)\left(\frac{\partial \mathrm{u}}{\partial \mathrm{z}}-\mathrm{f}^{\prime}\right)\right]\right|_{\mathrm{r}=\lambda_{\theta}-\mathrm{f}(\mathrm{z})+\mathrm{u}} \\
\left.\mathrm{v}\right|_{\mathrm{r}=\lambda_{\theta}-\mathrm{f}(\mathrm{z})+\mathrm{u}}=\frac{\partial \mathrm{u}}{\partial \mathrm{t}}+\left.\left(-\mathrm{f}^{\prime}+\frac{\partial \mathrm{u}}{\partial \mathrm{z}}\right) \mathrm{q}\right|_{\mathrm{r}=\lambda_{\theta}-\mathrm{f}(\mathrm{z})+\mathrm{u}}
\end{gathered}
$$

The definition of each variable in the Equation 1-5 has defined by Choy et al. (2012). The Equation 1-5 give sufficient relations to determine the field quantities $\mathrm{u}, \mathrm{v}$, $q$ and $p_{r}$ respectively.

\section{MATERIALS AND METHODS}

In this, we will investigate the amplitude modulation of weakly non-linear waves in a fluid-filled thin elastic tube with a stenosis whose non-dimensional governing equations are given in Equation 1-5. Considering the dispersion relation of the linearized field equations and the nature of the problem of concern, which is a boundary-value problem, the following stretched coordinates can be introduced:

$\xi=\varepsilon(\mathrm{z}-\lambda \mathrm{t}), \quad \tau=\varepsilon^{2} \mathrm{z}$

where, $\varepsilon$ is a small parameter measuring the weakness of nonlinearity and $\lambda$ is a constant to be determined from the solution. Introducing the following differential relations:

$\frac{\partial}{\partial \mathrm{t}}=\frac{\partial}{\partial \mathrm{t}}-\varepsilon \lambda \frac{\partial}{\partial \xi}, \frac{\partial}{\partial \mathrm{z}}=\frac{\partial}{\partial \mathrm{z}}+\varepsilon \frac{\partial}{\partial \xi}+\varepsilon^{2} \frac{\partial}{\partial \tau}$ 
and assuming that the field quantities may be expanding into asymptotic series of $\varepsilon$ as:

$$
\begin{aligned}
& \mathrm{u}=\varepsilon \mathrm{u}_{1}+\varepsilon^{2} \mathrm{u}_{2}+\varepsilon^{3} \mathrm{u}_{3}+\ldots, \\
& \mathrm{v}=\varepsilon \mathrm{v}_{1}+\varepsilon^{2} \mathrm{v}_{2}+\varepsilon^{3} \mathrm{v}_{3}+\ldots, \\
& \mathrm{q}=\varepsilon \mathrm{q}_{1}+\varepsilon^{2} \mathrm{q}_{2}+\varepsilon^{3} \mathrm{q}_{3}+\ldots, \\
& \overline{\mathrm{p}}=\overline{\mathrm{p}}_{0}+\varepsilon \overline{\mathrm{p}}_{1}+\varepsilon^{2} \overline{\mathrm{p}}_{2}+\varepsilon^{3} \overline{\mathrm{p}}_{3}+\ldots, \\
& \mathrm{p}_{\mathrm{r}}=\mathrm{p}_{\mathrm{r}_{0}}+\varepsilon \mathrm{p}_{\mathrm{r}_{1}}+\varepsilon^{2} \mathrm{p}_{\mathrm{r}_{2}}+\varepsilon^{3} \mathrm{p}_{\mathrm{r}_{3}}+\ldots \\
& \mathrm{h}(\mathrm{r})=\varepsilon^{2} \mathrm{~h}_{1}(\mathrm{r})+\varepsilon^{3} \mathrm{~h}_{2}(\mathrm{r})+\ldots
\end{aligned}
$$

where, u,v,q and $\mathrm{p}_{\mathrm{r}}$ are functions of the slow variables $(\xi \tau)$ as well as the fast variable $(\mathrm{z}, \mathrm{t})$.

Introducing (6)-(8) into the Equation 1-8, the following sets of differential Equations are obtained:

$\mathrm{O}(\varepsilon)$ Equations:

$\mathrm{p}_{\mathrm{r}_{1}}=\frac{\mathrm{m}}{\lambda_{\theta} \lambda_{\mathrm{z}}} \frac{\partial^{2} \mathrm{u}_{1}}{\partial \mathrm{t}^{2}}-\alpha_{0} \frac{\partial^{2} \mathrm{u}_{1}}{\partial \mathrm{z}^{2}}+\beta_{1} \mathrm{u}_{1}$,

$\frac{\partial \mathrm{v}_{1}}{\partial \mathrm{t}}+\frac{\partial \overline{\mathrm{p}}_{1}}{\partial \mathrm{r}}=0, \frac{\partial \mathrm{q}_{1}}{\partial \mathrm{t}}+\frac{\partial \overline{\mathrm{p}}_{1}}{\partial \mathrm{z}}=0, \frac{\partial \mathrm{v}_{1}}{\partial \mathrm{r}}+\frac{\mathrm{v}_{1}}{\mathrm{r}}+\frac{\partial \mathrm{q}_{1}}{\partial \mathrm{z}}=0$

and the boundary conditions:

$\left.\mathrm{v}_{1}\right|_{\mathrm{r}=\lambda_{\theta}}=\frac{\partial \mathrm{u}_{1}}{\partial \mathrm{t}},\left.\quad \overline{\mathrm{p}}_{1}\right|_{\mathrm{r}=\lambda_{\theta}}=\mathrm{p}_{\mathrm{r}_{1}}$

$\mathrm{O}\left(\varepsilon^{2}\right)$ Equations:

$$
\begin{aligned}
\mathrm{p}_{\mathrm{r}_{2}}= & \frac{\mathrm{m}}{\lambda_{\theta} \lambda} \frac{\partial^{2} \mathrm{u}_{2}}{\partial \mathrm{t}^{2}}-\alpha_{0} \frac{\partial^{2} \mathrm{u}_{2}}{\partial \mathrm{z}^{2}}+\beta_{1}\left(\mathrm{u}_{2}-\mathrm{h}_{1}\right) \\
& -\frac{2 \mathrm{~m} \lambda}{\lambda_{\theta} \lambda} \frac{\partial^{2} \mathrm{u}_{1}}{\partial \xi \partial \mathrm{t}}-2 \alpha_{0} \frac{\partial^{2} \mathrm{u}_{1}}{\partial \xi \partial \mathrm{z}}-\frac{\mathrm{m}}{\lambda_{\theta}^{2} \lambda_{\mathrm{z}}} \mathrm{u}_{1} \frac{\partial^{2} \mathrm{u}_{1}}{\partial \mathrm{t}^{2}} \\
& -\alpha_{1}\left(\frac{\partial \mathrm{u}_{1}}{\partial \mathrm{z}}\right)^{2}-\left(2 \alpha_{1}-\frac{\alpha_{0}}{\lambda_{\theta}}\right) \mathrm{u}_{1} \frac{\partial^{2} \mathrm{u}_{1}}{\partial \mathrm{z}^{2}}+\beta_{2} \mathrm{u}_{1}^{2} \\
\frac{\partial \mathrm{v}_{2}}{\partial \mathrm{t}}- & \lambda \frac{\partial \mathrm{v}_{1}}{\partial \xi}+\mathrm{v}_{1} \frac{\partial \mathrm{v}_{1}}{\partial \mathrm{r}}+\mathrm{q}_{1} \frac{\partial \mathrm{v}_{1}}{\partial \mathrm{z}}+\frac{\partial \overline{\mathrm{p}}_{2}}{\partial \mathrm{r}}=0 \\
\frac{\partial \mathrm{q}_{2}}{\partial \mathrm{t}}- & \lambda \frac{\partial \mathrm{q}_{1}}{\partial \xi}+\mathrm{v}_{1} \frac{\partial \mathrm{q}_{1}}{\partial \mathrm{r}}+\mathrm{q}_{1} \frac{\partial \mathrm{q}_{1}}{\partial \mathrm{z}}+\frac{\partial \overline{\mathrm{p}_{2}}}{\partial \mathrm{z}}+\frac{\partial \overline{\mathrm{p}_{1}}}{\partial \xi}=0 \\
\frac{\partial \mathrm{v}_{2}}{\partial \mathrm{r}}+ & \frac{\mathrm{v}_{2}}{\mathrm{r}}+\frac{\partial \mathrm{q}_{2}}{\partial \mathrm{z}}+\frac{\partial \mathrm{q}_{1}}{\partial \xi}=0
\end{aligned}
$$

and the boundary conditions:

$$
\begin{aligned}
& {\left.\left[\mathrm{u}_{1} \frac{\partial \mathrm{v}_{1}}{\partial \mathrm{r}}+\mathrm{v}_{2}\right]\right|_{\mathrm{r}=\lambda_{\theta}}=\frac{\partial \mathrm{u}_{2}}{\partial \mathrm{t}}-\lambda \frac{\partial \mathrm{u}_{1}}{\partial \xi}+\left.\frac{\partial \mathrm{u}_{1}}{\partial \mathrm{z}} \mathrm{q}_{1}\right|_{\mathrm{r}=\lambda_{\theta}}} \\
& {\left.\left[\mathrm{u}_{1} \frac{\partial \overline{\mathrm{p}}_{1}}{\partial \mathrm{r}}+\overline{\mathrm{p}}_{2}\right]\right|_{\mathrm{r}=\lambda_{\theta}}=\mathrm{p}_{\mathrm{r}_{2}} .}
\end{aligned}
$$

$\mathrm{O}\left(\varepsilon^{3}\right)$ Equations:

$$
\begin{aligned}
& \mathrm{p}_{\mathrm{r}_{3}}=\frac{\mathrm{m}}{\lambda_{\theta} \lambda_{\mathrm{z}}} \frac{\partial^{2} \mathrm{u}_{3}}{\partial \mathrm{t}^{2}}-\alpha_{0} \frac{\partial^{2} \mathrm{u}_{3}}{\partial \mathrm{z}^{2}}-\frac{2 \mathrm{~m} \lambda}{\lambda_{\theta} \lambda_{\mathrm{z}}} \frac{\partial^{2} \mathrm{u}_{2}}{\partial \xi \partial \mathrm{t}} \\
& -2 \alpha_{0} \frac{\partial^{2} u_{2}}{\partial \xi \partial z}-\alpha_{0}\left(\frac{\partial^{2} u_{1}}{\partial \xi^{2}}+2 \frac{\partial^{2} u_{1}}{\partial z \partial \tau}\right)+\frac{m \lambda^{2}}{\lambda_{\theta} \lambda_{z}} \frac{\partial^{2} u_{1}}{\partial \xi^{2}} \\
& +\beta_{1}\left(\mathrm{u}_{3}-\mathrm{h}_{2}\right)-\frac{\mathrm{m}}{\lambda_{\theta}^{2} \lambda_{\mathrm{z}}} \mathrm{u}_{1}\left(\frac{\partial^{2} \mathrm{u}_{2}}{\partial \mathrm{t}^{2}}-2 \lambda \frac{\partial^{2} \mathrm{u}_{1}}{\partial \xi \partial \mathrm{t}}\right) \\
& -\frac{\mathrm{m}}{\lambda_{\theta}^{2} \lambda_{\mathrm{z}}}\left(\mathrm{u}_{2}-\mathrm{h}_{1}\right) \frac{\partial^{2} \mathrm{u}_{1}}{\partial \mathrm{t}^{2}}-2 \alpha_{1} \frac{\partial \mathrm{u}_{1}}{\partial \mathrm{z}}\left(\frac{\partial \mathrm{u}_{2}}{\partial \mathrm{z}}+\frac{\partial \mathrm{u}_{1}}{\partial \xi}\right) \\
& -\left(2 \alpha_{1}-\frac{\alpha_{0}}{\lambda_{\theta}}\right) \mathrm{u}_{1}\left(\frac{\partial^{2} \mathrm{u}_{2}}{\partial \mathrm{z}^{2}}+2 \frac{\partial^{2} \mathrm{u}_{1}}{\partial \mathrm{z} \partial \xi}\right) \\
& -\left(2 \alpha_{1}-\frac{\alpha_{0}}{\lambda_{\theta}}\right)\left(\mathrm{u}_{2}-\mathrm{h}_{1}\right) \frac{\partial^{2} \mathrm{u}_{1}}{\partial \mathrm{z}^{2}}+2 \beta_{2} \mathrm{u}_{1}\left(\mathrm{u}_{2}-\mathrm{h}_{1}\right) \\
& +\frac{\mathrm{m}}{\lambda_{\theta}^{3} \lambda_{\mathrm{z}}} \mathrm{u}_{1}^{2} \frac{\partial^{2} \mathrm{u}_{1}}{\partial \mathrm{t}^{2}}-\left(\alpha_{2}-\frac{\alpha_{1}}{\lambda_{\theta}}\right) \mathrm{u}_{1}\left(\frac{\partial \mathrm{u}_{1}}{\partial \mathrm{z}}\right)^{2} \\
& -\left(\alpha_{2}-\frac{2 \alpha_{1}}{\lambda_{\theta}}+\frac{\alpha_{0}}{\lambda_{\theta}^{2}}\right) \mathrm{u}_{1}^{2} \frac{\partial^{2} \mathrm{u}_{1}}{\partial \mathrm{z}^{2}}+\beta_{3} \mathrm{u}_{1}^{3} \\
& -3\left(\gamma_{1}-\frac{\alpha_{0}}{2}\right) \mathrm{u}_{1}\left(\frac{\partial \mathrm{u}_{1}}{\partial \mathrm{z}}\right)^{2} \frac{\partial^{2} \mathrm{u}_{1}}{\partial \mathrm{z}^{2}} \\
& \frac{\partial \mathrm{v}_{3}}{\partial \mathrm{t}}-\lambda \frac{\partial \mathrm{v}_{2}}{\partial \xi}+\mathrm{v}_{1} \frac{\partial \mathrm{v}_{2}}{\partial \mathrm{r}}+\mathrm{v}_{2} \frac{\partial \mathrm{v}_{1}}{\partial \mathrm{r}}+\mathrm{q}_{1} \frac{\partial \mathrm{v}_{2}}{\partial \mathrm{z}}+\mathrm{q}_{1} \frac{\partial \mathrm{v}_{1}}{\partial \xi} \\
& +\mathrm{q}_{2} \frac{\partial \mathrm{v}_{1}}{\partial \mathrm{z}}+\frac{\partial \overline{\mathrm{p}}_{3}}{\partial \mathrm{r}}-\overline{\mathrm{v}}\left[\frac{\partial^{2} \mathrm{v}_{1}}{\partial \mathrm{r}^{2}}+\frac{1}{\mathrm{r}} \frac{\partial \mathrm{v}_{1}}{\partial \mathrm{r}}-\frac{\mathrm{v}_{1}}{\mathrm{r}^{2}}+\frac{\partial^{2} \mathrm{v}_{1}}{\partial \mathrm{z}^{2}}\right]=0 \text {, } \\
& \frac{\partial \mathrm{q}_{3}}{\partial \mathrm{t}}-\lambda \frac{\partial \mathrm{q}_{2}}{\partial \xi}+\mathrm{v}_{1} \frac{\partial \mathrm{q}_{2}}{\partial \mathrm{r}}+\mathrm{v}_{2} \frac{\partial \mathrm{q}_{1}}{\partial \mathrm{r}}+\mathrm{q}_{1} \frac{\partial \mathrm{q}_{2}}{\partial \mathrm{z}}+\mathrm{q}_{1} \frac{\partial \mathrm{q}_{1}}{\partial \xi} \\
& +\mathrm{q}_{2} \frac{\partial \mathrm{q}_{1}}{\partial \mathrm{z}}+\frac{\partial \overline{\mathrm{p}}_{3}}{\partial \mathrm{z}}+\frac{\partial \overline{\mathrm{p}}_{2}}{\partial \xi}+\frac{\partial \overline{\mathrm{p}}_{1}}{\partial \mathrm{r}}-\overline{\mathrm{v}}\left[\frac{\partial^{2} \mathrm{q}_{1}}{\partial \mathrm{r}^{2}}+\frac{1}{\mathrm{r}} \frac{\partial \mathrm{q}_{1}}{\partial \mathrm{r}}+\frac{\partial^{2} \mathrm{q}_{1}}{\partial \mathrm{z}^{2}}\right]=0 \\
& \frac{\partial \mathrm{v}_{3}}{\partial \mathrm{r}}+\frac{\mathrm{v}_{3}}{\mathrm{r}}+\frac{\partial \mathrm{q}_{3}}{\partial \mathrm{z}}+\frac{\partial \mathrm{q}_{2}}{\partial \xi}+\frac{\partial \mathrm{q}_{1}}{\partial \tau}=0
\end{aligned}
$$

and the boundary conditions:

$$
\begin{aligned}
& {\left.\left[\frac{1}{2} \mathrm{u}_{1}^{2} \frac{\partial^{2} \mathrm{v}_{1}}{\partial \mathrm{r}^{2}}+\left(\mathrm{u}_{2}-\mathrm{h}_{1}\right) \frac{\partial \mathrm{v}_{1}}{\partial \mathrm{r}}+\mathrm{u}_{1} \frac{\partial \mathrm{v}_{2}}{\partial \mathrm{r}}+\mathrm{v}_{3}\right]\right|_{\mathrm{r}=\lambda_{\theta}}} \\
& =\frac{\partial \mathrm{u}_{3}}{\partial \mathrm{t}}-\lambda \frac{\partial \mathrm{u}_{2}}{\partial \xi}+\frac{\partial \mathrm{u}_{1}}{\partial \mathrm{z}} \\
& {\left.\left[\mathrm{u}_{1} \frac{\partial \mathrm{q}_{1}}{\partial \mathrm{r}}+\mathrm{q}_{2}\right]\right|_{\mathrm{r}=\lambda_{\theta}}} \\
& +\left.\left[\frac{\partial \mathrm{u}_{2}}{\partial \mathrm{z}}+\frac{\partial \mathrm{u}_{1}}{\partial \xi}\right] \mathrm{q}_{1}\right|_{\mathrm{r}=\lambda_{\theta}}
\end{aligned}
$$




$$
\begin{aligned}
& {\left.\left[\begin{array}{l}
\frac{1}{2} \mathrm{u}_{1}^{2} \frac{\partial^{2} \overline{\mathrm{p}}_{1}}{\partial \mathrm{r}^{2}}+\left(\mathrm{u}_{2}-\mathrm{h}_{1}\right) \frac{\partial \overline{\mathrm{p}}_{1}}{\partial \mathrm{r}}+\mathrm{u}_{1} \\
\frac{\partial \overline{\mathrm{p}}_{2}}{\partial \mathrm{r}}+\overline{\mathrm{p}}_{3}-2 \mathrm{v} \frac{\partial \mathrm{v}_{1}}{\partial \mathrm{r}}
\end{array}\right]\right|_{\mathrm{r}=\lambda_{\theta}} .} \\
& =\mathrm{p}_{\mathrm{r}_{3}} .
\end{aligned}
$$

Here, the coefficients of $\alpha_{0}, \alpha_{1}, \alpha_{2}, \beta_{0}, \beta_{1}, \beta_{2}, \beta_{3}$ and $\gamma_{1}$ are defines by:

$\alpha_{0}=\frac{1}{\lambda_{\theta}} \frac{\partial \Sigma}{\partial \lambda_{z}}, \alpha_{1}=\frac{1}{2 \lambda_{\theta}} \frac{\partial^{2} \Sigma}{\partial \lambda_{\theta} \partial \lambda_{z}}, \alpha_{2}=\frac{1}{2 \lambda_{\theta}} \frac{\partial^{3} \Sigma}{\partial \lambda_{\theta}^{2} \partial \lambda_{z}}$,

$\gamma_{1}=\frac{\lambda_{\mathrm{z}}}{2 \lambda_{\theta}} \frac{\partial^{2} \Sigma}{\partial \lambda_{\mathrm{z}}^{2}}, \beta_{0}=\frac{1}{\lambda_{\theta} \lambda_{\mathrm{z}}} \frac{\partial \Sigma}{\partial \lambda_{\theta}}$

$\beta_{1}=\frac{1}{\lambda_{\theta} \lambda_{z}} \frac{\partial^{2} \Sigma}{\partial \lambda_{\theta}^{2}}-\frac{\beta_{0}}{\lambda_{\theta}}, \beta_{2}=\frac{1}{2 \lambda_{\theta} \lambda_{z}} \frac{\partial^{3} \Sigma}{\partial \lambda_{\theta}^{3}}-\frac{\beta_{1}}{\lambda_{\theta}}$,

$\beta_{3}=\frac{1}{6 \lambda_{\theta} \lambda_{z}} \frac{\partial^{4} \Sigma}{\partial \lambda_{\theta}^{4}}-\frac{\beta_{2}}{\lambda_{\theta}}$.

Equation 15 is defined through series expansion of the stretch ratios, $\lambda_{1}$ and $\lambda_{2}$ which read Equation 16:

$$
\begin{aligned}
\lambda_{1}= & \lambda_{\mathrm{z}}\left[1+\left\{\varepsilon \frac{\partial \mathrm{u}_{1}}{\partial \mathrm{z}}+\varepsilon^{2}\left[\frac{\partial \mathrm{u}_{2}}{\partial \mathrm{z}}+\frac{\partial \mathrm{u}_{1}}{\partial \xi}\right]\right.\right. \\
& \left.\left.+\varepsilon^{3}\left[\frac{\partial \mathrm{u}_{3}}{\partial \mathrm{z}}+\frac{\partial \mathrm{u}_{2}}{\partial \xi}+\frac{\partial \mathrm{u}_{1}}{\partial \tau}\right]\right\}^{2}\right]^{1 / 2} \\
\lambda_{2}= & \lambda_{\theta}+\varepsilon \mathrm{u}_{1}+\varepsilon^{2}\left[\mathrm{u}_{2}-\mathrm{h}_{1}\right]+\varepsilon^{3}\left[\mathrm{u}_{3}-\mathrm{h}_{2}\right] .
\end{aligned}
$$

By solving the sets of differential Equation 9-14, we obtain the following dissipative nonlinear Schrodinger Equation with variable coefficient:

$$
\mathrm{i} \frac{\partial \mathrm{U}}{\partial \tau}+\mu_{1} \frac{\partial^{2} \mathrm{U}}{\partial \xi^{2}}+\mu_{2}|\mathrm{U}|^{2} \mathrm{U}+\mu_{3} \mathrm{~h}_{1}(\tau) \mathrm{U}+\mathrm{i} \mu_{4} \mathrm{U}=0
$$

where, the coefficients $\mu, \mu_{1}, \mu_{2}, \mu_{3}$ and $\mu_{4}$ are defined by:

$$
\begin{aligned}
\mu= & 00\left[2 \alpha_{0} \mathrm{k}+\frac{\omega^{2}}{\mathrm{k}} \lambda_{\theta}\left\{\mathrm{f}_{0}^{2}-1\right\}\right]^{-1}, \\
\mu_{1}=\mu & \left\{-3 \lambda^{2}\left[\frac{\mathrm{f}_{0}}{\mathrm{k}}+\frac{\mathrm{m}}{\lambda_{\theta} \lambda_{\mathrm{z}}}\right]\right. \\
+ & \frac{\omega^{2}}{2 \mathrm{k}^{2}}\left[4 \frac{\lambda_{\theta} \lambda \mathrm{k}}{\omega} \mathrm{f}_{0}^{2}-\lambda_{\theta} \mathrm{f}_{0}^{2}-\lambda_{\theta}\right]
\end{aligned}
$$

$$
\begin{aligned}
& \left.+2 \lambda\left[\frac{\mathrm{m} \omega}{\lambda_{\mathrm{z}}} \mathrm{f}_{0}+2 \frac{\mathrm{k}}{\omega} \alpha_{0}\right]-\alpha_{0}\left[1+2 \mathrm{k} \lambda_{\theta} \mathrm{f}_{0}\right]\right\} \\
& \mu_{2}=\mu\left\{\omega ^ { 2 } \left[4 \frac{\lambda}{\omega \lambda_{\theta}^{2}} \mathrm{f}_{0}+6 \mathrm{kf}_{0}+\frac{3}{\mathrm{k} \lambda_{\theta}^{2}} \mathrm{f}_{0}-\frac{1}{2 \lambda_{\theta}}\right.\right. \\
& \left.-\frac{13}{2 \lambda_{\theta}} \mathrm{f}_{0}^{2}-4 \mathrm{kf}_{0}^{2} \mathrm{~F}_{0}+\frac{2}{\lambda_{\theta}} \mathrm{f}_{0} \mathrm{~F}_{0}\right]-5 \frac{\alpha_{1}}{\lambda_{\theta}} \mathrm{k}^{2} \\
& +3\left[\beta_{3}-\frac{\beta_{1}}{\lambda_{\theta}^{2}}\right]+2 \alpha_{2} \mathrm{k}^{2}-\frac{3}{2} \alpha_{0} \mathrm{k}^{4}+3 \gamma_{1} \mathrm{k}^{4} \\
& +\left[4 \frac{\lambda \omega}{\lambda_{\theta}} \mathrm{f}_{0}+\omega^{2}\left\{\mathrm{f}_{0}^{2}-\frac{2}{\mathrm{k} \lambda_{\theta}} \mathrm{f}_{0}-1\right\}+2 \beta_{2}\right. \\
& \left.+2 \alpha_{1} \mathrm{k}^{2}+\frac{\beta_{1}}{\lambda_{\theta}}\right]\left[2 \lambda^{2}-\lambda_{\theta} \beta_{1}\right]^{-1} \\
& \times\left[4 \lambda \omega \mathrm{f}_{0}-2 \frac{\lambda^{2}}{\lambda_{\theta}}+2 \beta_{1}+2 \alpha_{1} \lambda_{\theta} \mathrm{k}^{2}+2 \beta_{2} \lambda_{\theta}\right. \\
& \left.+\omega^{2}\left\{\lambda_{\theta} \mathrm{f}_{0}^{2}-\lambda_{\theta}-\frac{2}{\mathrm{k}} \mathrm{f}_{0}\right\}\right] \\
& +\left[\omega^{2}\left\{\mathrm{f}_{0}^{2}-\frac{6}{\mathrm{k} \lambda_{\theta}} \mathrm{f}_{0}-3+4 \mathrm{f}_{0} \mathrm{~F}_{0}\right\}+2 \beta_{2}\right. \\
& \left.+6 \alpha_{1} \mathrm{k}^{2}+5 \frac{\beta_{1}}{\lambda_{\theta}}\right]\left[-3 \beta_{1}+4 \frac{\omega^{2}}{\mathrm{k}} \mathrm{f}_{0}-2 \frac{\omega^{2}}{\mathrm{k}} \mathrm{F}_{0}\right]^{-1} \\
& \times\left[\omega^{2}\left\{\frac{3}{2}-\frac{1}{2} \mathrm{f}_{0}^{2}+\frac{1}{\mathrm{k} \lambda_{\theta}} \mathrm{f}_{0}\right\}-3 \alpha_{1} \mathrm{k}^{2}\right. \\
& \left.\left.+\frac{\omega^{2}}{\mathrm{k}}\left\{\frac{1}{\lambda_{\theta}}-2 \mathrm{kf}_{0}\right\} \mathrm{F}_{0}-\beta_{2}-\frac{\beta_{1}}{\lambda_{\theta}}\right]\right\} \text {, } \\
& \mu_{3}=\mu\left\{-\frac{\lambda_{\theta} \beta_{1}}{2 \lambda^{2}-\lambda_{\theta} \beta_{1}}\left[\omega^{2}\left\{\mathrm{f}_{0}^{2}-\frac{2}{\mathrm{k} \lambda_{\theta}} \mathrm{f}_{0}-1\right\}+2 \beta_{2}\right.\right. \\
& \left.+\frac{\beta_{1}}{\lambda_{\theta}}+4 \frac{\omega \lambda}{\lambda_{\theta}} \mathrm{f}_{0}+2 \alpha_{1} \mathrm{k}^{2}\right]-2\left(\alpha_{1} \mathrm{k}^{2}+\beta_{2}\right) \\
& \left.+\omega^{2}\left\{\frac{2}{\mathrm{k} \lambda_{\theta}} \mathrm{f}_{0}-\mathrm{f}_{0}^{2}+1\right\}-\frac{\beta_{1}}{\lambda_{\theta}}\right\} \text {, } \\
& \mu_{4}=\frac{2 \omega \mathrm{v}\left[\mathrm{kf}_{0}-\frac{1}{\lambda_{\theta}}\right]}{2 \mathrm{k} \alpha_{0}+\frac{\omega^{2} \lambda_{\theta}}{\mathrm{k}}\left(\mathrm{f}_{0}^{2}-1\right)} \text {. }
\end{aligned}
$$

The group velo city, $\lambda$ is defined as given in the Equation 19:

$$
\lambda=\frac{\omega^{2} \lambda_{\theta}\left[\mathrm{f}_{0}^{2}-1\right]+2 \alpha_{0} \mathrm{k}^{2}}{2 \omega\left[\mathrm{f}_{0}+\frac{\mathrm{mk}}{\lambda_{\theta} \lambda_{\mathrm{z}}}\right]}
$$


And the dispersion relation is read as given in the Equation 20:

$$
\frac{\omega^{2} \mathrm{f}_{0}}{\mathrm{k}}+\frac{\mathrm{m} \omega^{2}}{\lambda_{\theta} \lambda_{\mathrm{z}}}-\alpha_{0} \mathrm{k}^{2}-\beta_{1}=0
$$

Refering Equation 18, we have defined the following function as given in the Equation 21:

$$
\mathrm{f}_{0}=\frac{\mathrm{I}_{0}\left(\mathrm{k} \lambda_{\theta}\right)}{\mathrm{I}_{1}\left(\mathrm{k} \lambda_{\theta}\right)}, \quad \mathrm{F}_{0}=\frac{\mathrm{I}_{0}\left(2 \mathrm{k} \lambda_{\theta}\right)}{\mathrm{I}_{1}\left(2 \mathrm{k} \lambda_{\theta}\right)}
$$

where, $\mathrm{I}_{\mathrm{n}}$ referred as modified bessel's function of order $\mathrm{n}$.

For the governing NLS type Equation 17, it is For the governing NLS type Equation 17, it is found that the dissipative term, $i \mu_{4} U$ is caused by the viscous effect. Therefore, in the absence of viscous effect, the dissipative NLS equation with variable coefficient (17) will be reduced to the NLS equation with variable coefficient. Besides, noted that the presence of stenosis causes the variable coefficient term $\mu_{3} h_{1}(\tau) \mathrm{U}$ in the Equation 17. In other words, without the presence of stenosis and viscous effect in the physical model, the dissipative NLS equation with variable coefficient will be reduced to the standard NLS equation.

By solving Equation 17, the progressive wave solution for the dissipative NLS equation with variable coefficient is obtained as:

$$
\begin{aligned}
& \mathrm{U}(\xi, \tau)=\mathrm{a}_{0} \mathrm{e}^{-\frac{2}{3} \mu_{4} \tau} \tanh \left[\begin{array}{l}
\left(-\frac{\mu_{2}}{2 \mu_{1}}\right)^{1 / 2} \\
\mathrm{a}_{0} \mathrm{e}^{-\frac{2}{3} \mu_{4} \tau}\left(\xi-2 \mu_{1} \mathrm{~K} \tau\right)
\end{array}\right]
\end{aligned}
$$

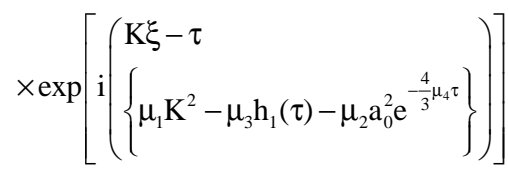

Inserting Equation (6) into Equation (22) yields given in the Equation 23:

$$
\begin{aligned}
\mathrm{U}(\mathrm{t}, \mathrm{z})= & \mathrm{a}_{0} \mathrm{e}^{-\frac{2}{3} \mu_{3} \varepsilon^{2} \mathrm{z}} \tanh \left[\left(-\frac{\mu_{2}}{2 \mu_{1}}\right)^{1 / 2} \mathrm{a}_{0} \mathrm{e}^{-\frac{2}{3} \mu_{3} \varepsilon^{2} \mathrm{z}}(\varepsilon \mathrm{z}-\varepsilon \lambda \mathrm{t}\right. \\
& \left.\left.-2 \mu_{1} \mathrm{~K} \varepsilon^{2} \mathrm{z}\right)\right] \exp \left[\mathrm { i } \left(\mathrm{K} \varepsilon(\mathrm{z}-\lambda \mathrm{t})-\varepsilon^{2} \mathrm{z}\left\{\mu_{1} \mathrm{~K}^{2}\right.\right.\right. \\
& \left.\left.\left.-\mu_{3} \mathrm{~h}_{1}\left(\varepsilon^{2} \mathrm{z}\right)-\mu_{2} \mathrm{a}_{0}^{2} \mathrm{e}^{-\frac{4}{3} \mu_{3} \varepsilon^{2} \mathrm{z}}\right\}\right]\right)
\end{aligned}
$$

\section{RESULTS}

In order to observe the wave modulation in the stenosed tube, one has to know the values of coefficients $\mu_{1}, \mu_{2}, \mu_{3}$ and $\mu_{4}$. To obtain values of these coefficients, one can referred Kim et al. (2010). The modulus of the solution of the dissipative NLS equation with variable coefficient (22) versus space, $\tau$ and travelling wave profile, $\xi$ are displayed in Fig. 1 and 2, respectively. Figure 3 illustrates the variations of the radial displacement, $\mathrm{U}(\mathrm{t}, \mathrm{z})$ with space $\mathrm{z}$ when viscosity of the fluid. $\mathrm{V}=100$.

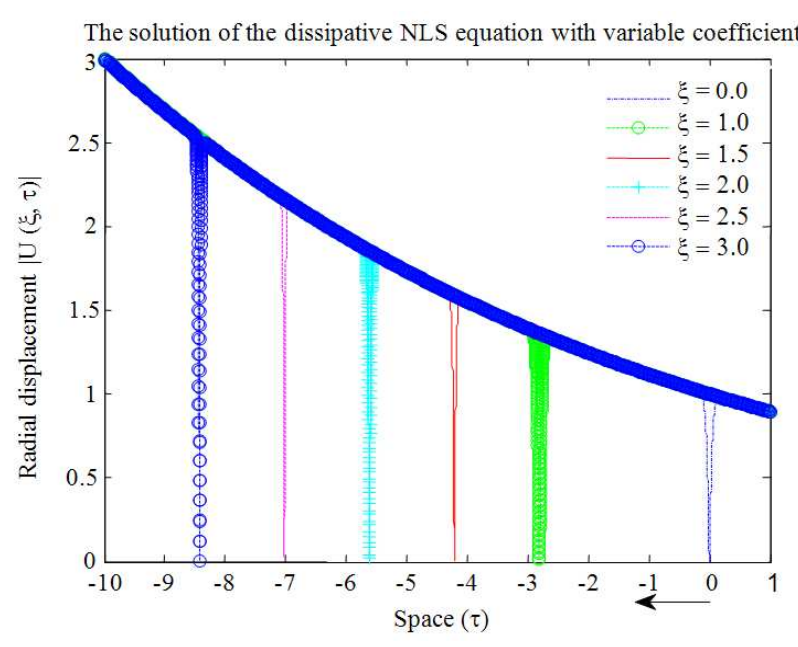

Fig. 1. The modulus of the solution (22) versus space, $\tau$ for different travelling wave profile, $\xi$ at $\delta=0.30$

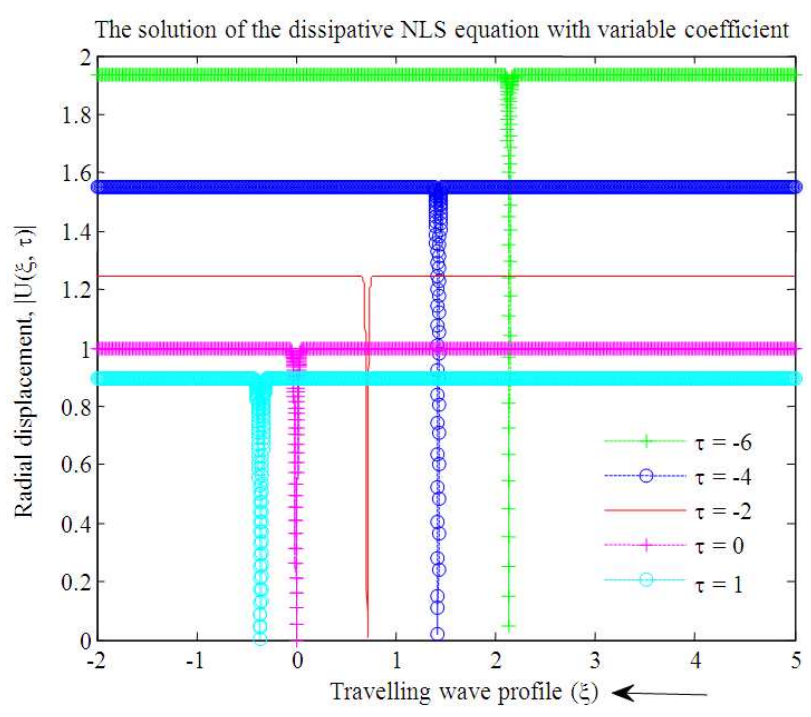

Fig. 2. The modulus of the solution (22) versus travelling wave profile, $\xi$ for different space, $\tau$ at $\delta=0.30$ 


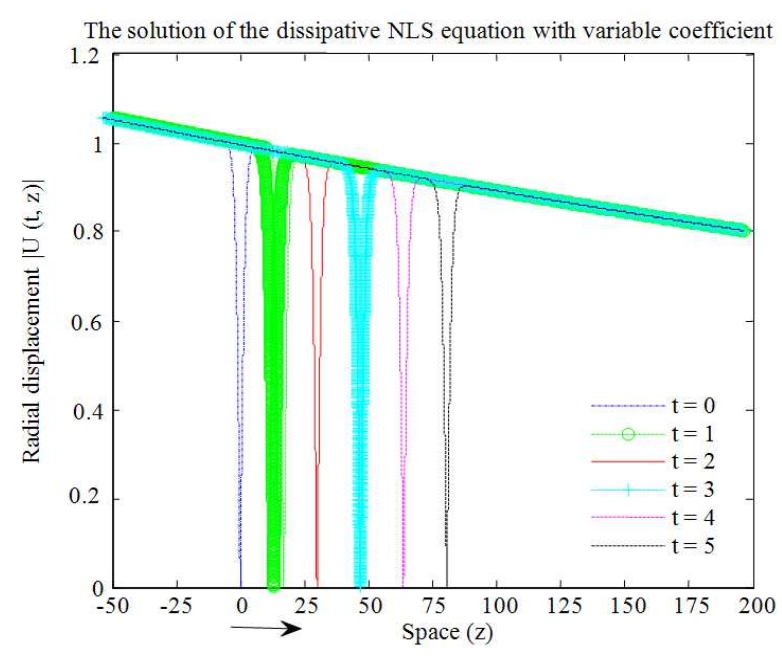

Fig. 3. The modulus of the solution (23) versus space, $\mathrm{z}$ for different time, $\mathrm{t}$ at $\delta=0.30$ when $\mathrm{v}=100$

\section{DISCUSSION}

The first and the second graphs reveal the downward bell-shaped wave travels to the left as travelling wave profile and space increase, respectively. Notice that in Fig. 2 due to the viscous effect, the wave propagates with decreasing wave amplitude when the space increases. As can be seen from the third figure, the viscous effect of the fluid causes the downward bellshaped wave propagates to the right with decreasing wave amplitude as time, $t$ increases. The big nonlinearity effect leads the solitary wave performs a narrower downward bell-shaped wave.

\section{CONCLUSION}

We presented the modulation of nonlinear waves in a prestressed thin-walled elastic tube with a symmetrical stenosis filled with the Newtonian fluid. The governing equation is obtained as the dissipative Nonlinear Schrodinger (NLS) equation with variable coefficient.

Through the model of Newtonian fluid in the stenosed artery, it is seen that the wave propagates to the right with decreasing wave amplitude when the time, $t$ increases.

\section{REFERENCES}

Akgun, G. and H. Demiray, 2000. Modulation of nonlinear axial and transverse waves in a fluid-filled thin elastic tube. Int. J. Non-Linear Mech., 35: 597611. DOI: 10.1016/S0020-7462(99)00044-X
Akgun, G. and H. Demiray, 2001. Interactions of nonlinear acoustic waves in a fluid-filled elastic tube. Int. J. Eng. Sci., 39: 563-581. DOI: 10.1016/S0020-7225(00)00057-4

Choy, Y.Y., C.T. Ong and K.G., Tay, 2012. NLS equation with a variable coefficient in a stenosed elastic tube filled with an average inviscid fluid. Math. J. Univ. Teknol. Malaysia, 28: 1-13.

Demiray, H., 1997. Nonlinear wave modulation in a prestressed thin elastic tube filled with an inviscid fluid. IMA J. Applied Mathem., 59: 165-181. DOI: 10.1093/imamat/59.2.165

Demiray, H., 1998. Nonlinear wave modulation in a fluid-filled thick elastic tube. Int. J. Eng. Sci., 36: 1061-1082. DOI: 10.1016/S0020-7225 (98)00008-1

Demiray, H., 2001. Modulation of non-linear waves in a viscous fluid contained in an elastic tube. Int. J. Non-Linear Mech., 36: 649-661. DOI: 10.1016/S0020-7462(00)00029-9

Demiray, H., 2003. Contributions of higher order terms to nonlinear waves in fluid-filled elastic tubes: Strongly dispersive case. Int. J.Eng. Sci., 4: $1387-$ 1403. DOI: $10.1016 / \mathrm{S} 0020-7225(03) 00039-9$

Gaik, T.K. and H. Demiray, 2008. Forced korteweg-de vries-burgers equation in an elastic tube filled with a variable viscosity fluid. Chaos, Solitons Fractals, 38: 1134-1145. DOI: 10.1016/j.chaos.2007.02.005

Kim, G.T., 2006. Forced korteweg-de vries equation in an elastic tube with an inviscid fluid. Int. J. Eng. Sci., 44: 621-632. DOI: 10.1016/j.ijengsci. 2006.04.008

Kim, G.T., Y.C. Yaan, T.O. Chee and H. Demiray, 2010. Dissipative non-linear schrodinger equation with variable coefficient in a stenosed elastic tube filled with a viscous fluid. Int. J. Eng. Sci. Technol., 2: 708-723.

Ravindran, R. and P. Prasad, 1979. A mathematical analysis of nonlinear waves in a fluid-filled viscoelastic tube. Acta Mech., 31: 253-280. DOI: 10.1007/BF01176853

Tay, K.G., C.T. Ong and M.N.Mohamad, 2007. Forced perturbed korteweg-de vries equation in an elastic tube filled with a viscous fluid. Int. J. Eng. Sci., 45: 339-349. DOI: 10.1016/j.ijengsci.2007.03.017 